\title{
Pterygomaxillary Disjunction and its Influence on the Result of Surgically Assisted Maxillary Expansion: A Systematic Review and Meta-analysis
}

Bruno da Silva Mesquita ${ }^{1}$, Belmiro Cavalcanti do Egito Vasconcelos ${ }^{2}$, Sandra Lucia Dantas de Moraes ${ }^{3}$, Cleidiel Aparecido Araújo Lemos ${ }^{4}$, Jéssica Marcela de Luna Gomes ${ }^{5}$, Eduardo Piza Pellizzer ${ }^{6}$, Emanuel Sávio de Souza Andrade ${ }^{7}$

\begin{abstract}
Aim: The aim of this study was to evaluate the available evidence to identify the influence of pterygomaxillary disjunction on the result of surgically assisted maxillary expansion.

Background: LeFort I type osteotomy with disjunction of the pterygomaxillary suture is a procedure widely used in maxillofacial surgery. However, the need for its performance during surgically assisted maxillary expansions has been discussed in literature, since serious complications can be caused during this stage.

Review results: Systematic review of articles was performed using three databases (PubMed, Web of Science, and Cochrane) published until May 2019. After applying the selection criteria, five articles were included in the systematic review, with a total of 141 patients. Meta-analysis showed the absence of significant difference between intervention and control groups in the preoperative period (standardized mean difference $=-0.28$; confidence interval, $\mathrm{Cl} 95 \%=-0.81,0.26 ; p=0.31$ ) and postoperative period (standardized mean difference $=-0.12 ; 95 \% \mathrm{Cl}=-0.65,0.42$; $p=0.66)$. In general, the heterogeneity of statistical estimates was low $\left(I^{2}=0 \%\right)$.

Conclusion: No statistically significant difference was observed between control group (without pterygomaxillary disjunction) and intervention group (with pterygomaxillary disjunction).

Clinical significance: Based on the data analyzed in this systematic review, it could be concluded that pterygomaxillary disjunction is not a mandatory step to achieve satisfactory maxillary expansion. Thus, not performing pterygomaxillary disjunction can prevent complications and reduce surgical time.

Keywords: LeFort I osteotomy, Maxilla, Systematic review.

The Journal of Contemporary Dental Practice (2020): 10.5005/jp-journals-10024-2857
\end{abstract}

\section{INTRODUCTION}

Surgically assisted maxillary expansion is a technique widely used in maxillofacial surgery. It is used in the treatment of transverse maxillary bone deficiencies, providing well-defined and reliable surgical protocol. LeFort I type osteotomy with separation of the midpalatal suture and pterygomaxillary disjunction is the most widely accepted and used technique for this surgery. However, some authors propose the nondisjunction of the pterygomaxillary suture, especially for small expansions (less than $6 \mathrm{~mm}$ ), ${ }^{1}$ since studies have shown that most complications (such as major hemorrhages and pseudoaneurysms) of LeFort I type osteotomy are related to this procedure. Likewise, trans-surgical complications related to nondisjunction (such as skull base fracture) occur mainly when there is a need for maxillary mobilization, which does not apply to expansions. ${ }^{2}$

Based on the above, the question about the significant difference in performing disjunction still seeks an answer in literature. Although arousing the interest of researchers, published studies are inconclusive. ${ }^{3}$ One reason for this is the lack of more standardized protocols, especially with regard to expansion measurement. Thus, computed tomography proved to be the most reliable method, with the best methodological basis, due to its accuracy. In addition, it provides reproducible standardization and eliminates possible manual measurement errors, such as those made on plaster models. ${ }^{4}$

Considering the current literature, the research question is as follows: are there new studies that homogeneously evaluate the influence of pterygomaxillary disjunction on surgically assisted
${ }^{1-4,7}$ Faculty of Dentistry, Department of Oral and Maxillofacial Surgery, University of Pernambuco, Camaragibe, Pernambuco, Brazil

${ }^{5,6}$ Faculty of Dentistry, Department of Dental Materials and Prosthesis, São Paulo State University, Araçatuba, São Paulo, Brazil

Corresponding Author: Bruno da Silva Mesquita, Faculty of Dentistry, Department of Oral and Maxillofacial Surgery, University of Pernambuco, Camaragibe, Pernambuco, Brazil, Phone: +55 (81) 31847661, e-mail: brunomesquitajpa@hotmail.com

How to cite this article: da Silva Mesquita B, do Egito Vasconcelos BC, de Moraes SLD, et al. Pterygomaxillary Disjunction and its Influence on the Result of Surgically Assisted Maxillary Expansion: A Systematic Review and Meta-analysis. J Contemp Dent Pract 2020;21(6):696-700.

Source of support: Scholarship awarded by the Coordination for the Improvement of Higher Education Personnel (CAPES)

Conflict of interest: None

maxillary expansion? The aim of this study was to evaluate the available evidence to identify the influence of pterygomaxillary disjunction on surgically assisted maxillary expansion.

\section{Materials and Methods}

\section{General Aspects}

This systematic review was conducted according to the guidelines of the Preferred Reporting Items for Systematic Reviews and MetaAnalyzes. ${ }^{5}$

(0) The Author(s). 2020 Open Access This article is distributed under the terms of the Creative Commons Attribution 4.0 International License (https://creativecommons. org/licenses/by-nc/4.0/), which permits unrestricted use, distribution, and non-commercial reproduction in any medium, provided you give appropriate credit to the original author(s) and the source, provide a link to the Creative Commons license, and indicate if changes were made. The Creative Commons Public Domain Dedication waiver (http://creativecommons.org/publicdomain/zero/1.0/) applies to the data made available in this article, unless otherwise stated. 


\section{The PICOS}

The PICOS method (population, intervention, comparator test, outcomes, studies) was used to define the eligibility criteria for inclusion of studies in this systematic review. ${ }^{5}$ According to PICOS, the specific research question was the following: is there any difference in the results of different surgical techniques used in pterygomaxillary disjunction to perform surgically assisted maxillary expansion?

The various PICOS components were population-individuals with indication for surgically assisted maxillary expansion; intervention-surgically assisted maxillary expansion with disruption of the pterygomaxillary suture; control-surgically assisted maxillary expansion without disruption of the pterygomaxillary suture; result-posterior maxillary expansion; and secondary outcomes-anterior osteotomies (two or three segments) and distractors (bone or dental).

\section{Search Methodology}

A systematic search was conducted in PubMed (1966 to May 2019), Web of Science (1990 to May 2019), and Cochrane Library (1996 to May 2019) electronic databases for articles. The search period has been extended since the foundation of each database. Restrictions on the year, language, and status of publication were not applied. Relevant studies were identified using the following combination of key words: [(surgically assisted rapid palatal expansion OR surgically assisted rapid maxillary expansion OR Palatal Expansion Technique OR SARPE OR SARME) AND (separation pterygomaxillary OR pterygomaxillary disjunction OR pterygoid disjunction OR pterygoid plates)]. Manual cross-reference search of included studies was also conducted in order to identify any additional relevant studies.

\section{Selection Criteria}

The selection criteria for the inclusion of studies in this systematic review were clinical trials in humans in which participants underwent surgically assisted maxillary expansion performed with LeFort I type osteotomy in two segments with methodology that clearly compared, by tomography, the performance of the surgery with or without disjunction of the pterygomaxillary suture in the expansion in posterior region using molar teeth as reference. There were no language or year of publication (date) restrictions for the inclusion of studies in this review. Case reports, case series, letters to the editor, conference proceedings, and articles without access to the full text were excluded. Studies that included individuals with syndromes or anomalies were also excluded. The Kappa test was used to assess the agreement of each author regarding the inclusion of studies.

\section{Data Extraction}

Two reviewers (BSM and CAA) independently analyzed titles, abstracts, and results of studies, identifying all potentially relevant ones. Studies included in this phase were read in full and evaluated according to the eligibility criteria to be included in the final systematic review. Disagreements between reviewers were resolved through discussion to obtain consensus or by discussion with a third reviewer (BSM, CAA and ESSA). The following information was analyzed: study design, study population, indication for surgically assisted maxillary expansion, and outcome measures.

\section{Data Analysis}

A meta-analysis of continuous results was performed to estimate the effect on posterior maxillary expansion after intervention (with disjunction of the pterygomaxillary suture) compared to control (without disjunction of the pterygomaxillary suture). Differences in results were demonstrated by a forest plot graph, using the random effects model to determine the standardized mean difference, considering $95 \%$ confidence intervals and $p$ values. ${ }^{6,7}$ Statistical heterogeneity among studies was assessed using the $I^{2}$ statistics, which was classified as follows: low $\left(I^{2}<25 \%\right)$, moderate $\left(I^{2}=50 \%\right)$, and high $\left(I^{2}>75 \%\right) .{ }^{8}$ Publication bias was not evaluated because the funnel plot tests required at least 10 studies, a condition not met in our systematic review, ${ }^{9}$ which is something that would make the power of tests too low to distinguish the likelihood of real

Flowchart 1: Flowchart describing the study selection process
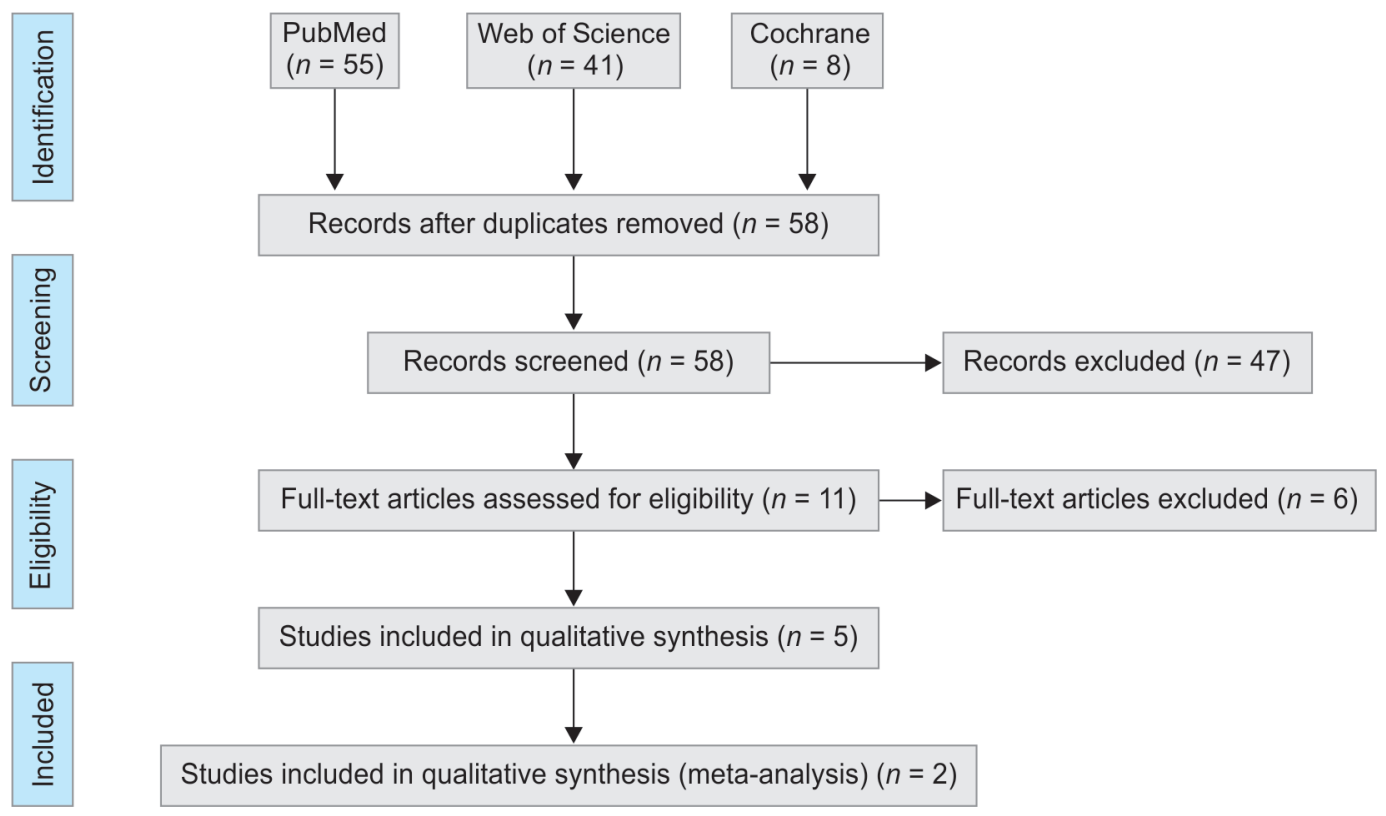
asymmetry. The Review Manager software version 5.3 (RevMan, Cochrane Collaboration) was used to perform all statistical analyzes.

\section{Review Results}

A total of 104 articles were selected in the initial search of the three databases (PubMed, Web of Science, and Cochrane). After excluding duplicates, the remaining 58 articles were selected. Of these, 47 studies were excluded after reading titles and abstracts. Thus, 11 articles were selected for full reading. According to the eligibility criteria, five studies ${ }^{10-14}$ were included in the systematic review with good agreement between reviewers $(\kappa=0.82)$. With qualitative analysis being carried out, the real potential for the inclusion of two articles ${ }^{10,12}$ in the meta-analysis was observed.

A summary of the study selection process, is shown in Flowchart 1. The complete evaluation of these studies (data extraction) is shown in Table 1.

\section{General Aspects}

The systematic review included a total of 141 individuals. The device most used in studies was the dental anchorage (Hyrax), which is an orthodontic device used for maxillary expansion. This device is cemented in premolar and first molar regions before maxillary expansion surgery and is activated after a latency period of 5-7 days. The study by Laudemann et al. ${ }^{11}$ was the only one that used the maxilla widening device (MWD). The daily activation protocol was similar in the five studies, with two activations and expansions ranging from 0.4 to $0.6 \mathrm{~mm} /$ day (Table 2 ).

Regarding the distances measured between molar teeth (intermolar distance), three studies ${ }^{11,12,14}$ observed posterior expansion in patients undergoing maxilla widening with pterygomaxillary disjunction. Four studies ${ }^{11-14}$ also demonstrated posterior expansion in patients undergoing maxilla widening without pterygomaxillary disjunction. In turn, Ferraro-Bezerra et al. ${ }^{10}$ did not observe expansion in the posterior region in any groups. Otherwise, a decrease of 0.1 and $2 \mathrm{~mm}$ was observed in groups with and without pterygomaxillary disjunction, respectively.

\section{Meta-analysis}

Only two studies ${ }^{10,12}$ had enough data to be included in the metaanalysis, which evidenced through their results the absence of significant difference between intervention and control groups in the preoperative period (standardized mean difference $=-0.28 ; \mathrm{Cl}$ $95 \%=-0.81,0.26 ; p=0.31$ ) and postoperative period (standardized mean difference $=-0.12 ; 95 \% \mathrm{Cl}=-0.65,0.42 ; p=0.66$ ) (Fig. 1). In general, the heterogeneity of statistical estimates was low $\left(I^{2}=0 \%\right)$.

\section{Discussion}

The use of pterygomaxillary disjunction does not seem to be necessary for successful maxillary expansion, since no statistically significant difference was observed between groups. Although the difference between pre- and postoperative measurements (delta) varied from -0.1 to $6.84 \mathrm{~mm}$, it is possible to perceive statistical equality when this distance is evaluated within the margin of error, considering the standard deviation of each study. However, this cannot be stated with complete certainty, despite the low heterogeneity in results presented here. Several studies have used different methodologies, which made it difficult to include them in the meta-analysis. Consequently, the construction of an objective and reliable conclusion was impaired.

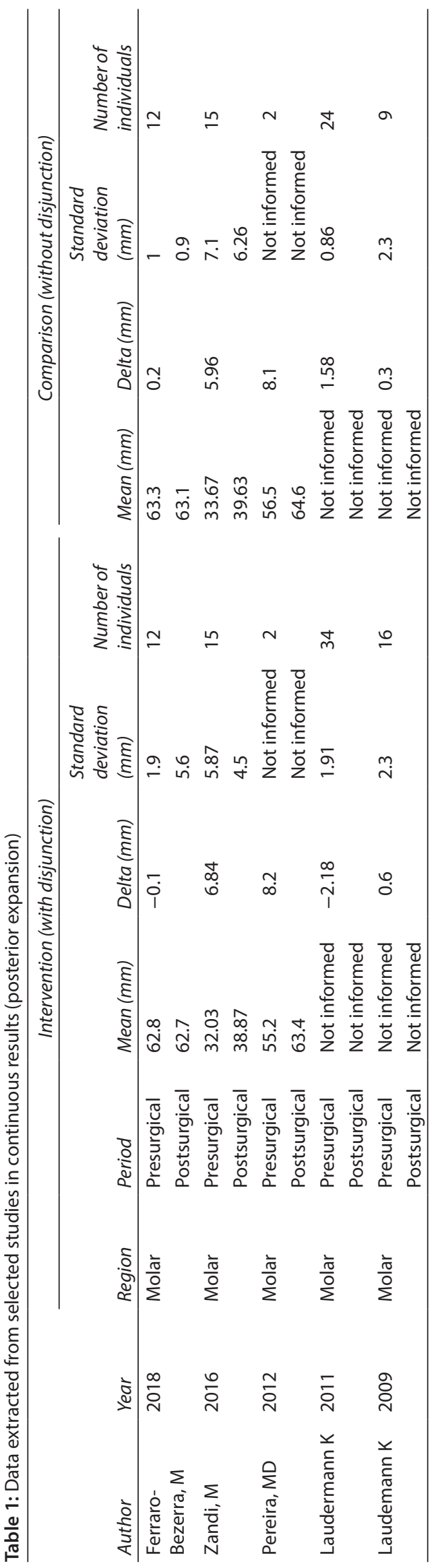


Table 2: Secondary data extracted

\begin{tabular}{lllll}
\hline Secondary information & & & \multicolumn{2}{c}{ Daily activations } \\
\cline { 4 - 5 } Author & & & 2 & 0.5 \\
\hline Ferraro-Bezerra, M & 2018 & Hyrax & 2 & $0.5-0.6$ \\
Zandi, M & 2016 & Hyrax & 2 & 0.4 \\
Pereira, MD & 2012 & Hyrax & 2 & $0.5-0.6$ \\
Laudemann K & 2011 & Hyrax & 2 & $0.5-0.6$ \\
Laudemann K & 2009 & Hyrax & 2 & $0.5-0.6$ \\
\hline
\end{tabular}

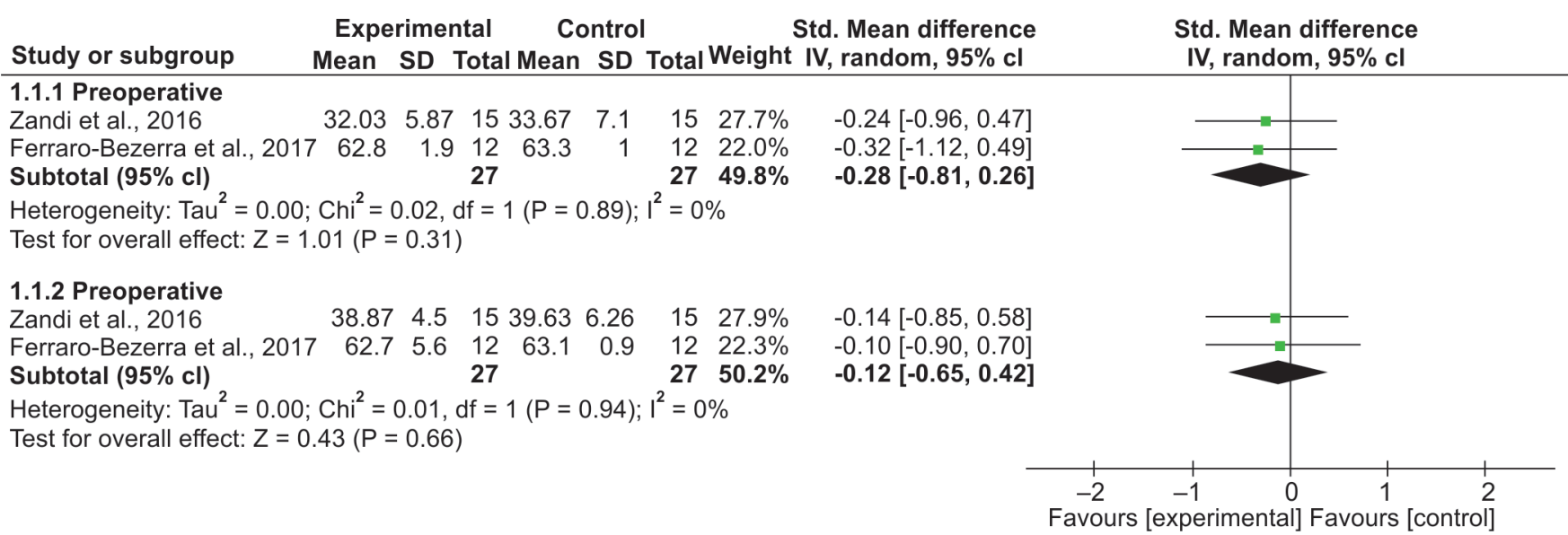

Fig. 1: Forest plot graph of estimates reported by studies considered eligible for this meta-analysis. Standardized mean differences of evaluated parameters and their respective $95 \%$ confidence intervals are represented by squares (each square represents one study). Diamonds represent the general average difference estimated from included studies

In addition, important variables such as the maximum expansion quantity were not mentioned in most studies, which may also have compromised the reliability of results. However, expansions close to or greater than $6 \mathrm{~mm}$ were equivalent in both groups. In this context, age was an important confounding factor, with the perception that posterior maxillary expansion without disjunction in patients under 20 years of age has more satisfactory results. $^{13}$

In 2018, Ferraro-Bezerra et al. ${ }^{10}$ observed significant expansion and crossbite corrections for both disjunction and non-disjunction groups, identifying greater expansion in the molar region (posterior) compared to the canine region (anterior). In this study, group treated without pterygomaxillary disjunction had expansion of $1 \mathrm{~mm}$ more in the molar region, compared to the group treated with pterygomaxillary disjunction. However, there was no bone expansion due to the fact that dental movements are induced during Hyrax activation, increasing the maxillary perimeter caused by teeth inclination, not bone expansion. ${ }^{15}$

Thus, the type of device seems to directly influence the result of surgically assisted maxillary expansions. The greatest preference was for devices with dental anchorage (Table 2). However, when analyzed, MWDs also showed variations in the results of expansions. In patients without pterygomaxillary disjunction, the MWD appeared to allow greater expansion in the anterior region when compared to the posterior region due to the fact that there is immobilization in the pterygomaxillary suture. ${ }^{13}$

In fact, in an "anatomical illustration", it seems obvious that the pterygomaxillary disjunction is essential for adequate maxillary expansion. ${ }^{16}$ This may explain why some studies tend to achieve greater expansion in the anterior region in the group without pterygomaxillary disjunction. Thus, pterygomaxillary disjunction can be indicated mainly in cases of posterior crossbite (or large expansions), which would facilitate bone widening in this region. ${ }^{16}$ However, we cannot confirm this result, since in our study, no significant differences were found between performance or not performance of pterygomaxillary disjunction. On the contrary, we agree with Vasconcelos et al. ${ }^{17}$ who contradicted this theory and observed greater posterior expansion in groups not subject to pterygomaxillary disjunction.

The present review is original and contributed to the advancement of scientific knowledge. However, it was not free of limitations. The articles varied mainly about sample size and methods used. The results of the meta-analysis should be analyzed with caution, due to the small number of studies. Associated variables such as age, type of device, and maximum expansion must be taken into account. For this, further clinical trials that can propose a clear, reliable, and easy-to-reproduce protocol should be carried out, which would provide for a future systematic review with greater evidence.

\section{Conclusion}

As there was no statistically significant difference between control group (without pterygomaxillary disjunction) and intervention group (with pterygomaxillary disjunction), not performing pterygomaxillary disjunction can prevent complications and reduce surgical time. 


\section{Clinical Significance}

Based on the data analyzed in this systematic review, it could be concluded that pterygomaxillary disjunction is not a mandatory step to achieve satisfactory maxillary expansion. From this point of view, a more conservative approach will have direct effects on postoperative recovery, with less pain and edema, without compromising on the final maxillary expansion result.

\section{References}

1. Esen A, Soganci E, Dolanmaz E, et al. Evaluation of stress by finite element analysis of the midface and skull base at the time of midpalatal osteotomy in models with or without pterygomaxillary dysjunction. Br J Oral Maxillofac Surg 2018;56(3):177-181. DOI: 10.1016/j.bjoms.2018.01.001.

2. Pereira MD, Koga AF, Prado GPR, et al. Complications from surgically assisted rapid maxillary expansion with HAAS and Hyrax expanders. J Craniofac Surg 2018;29(2):275-278. DOI: 10.1097/ SCS.0000000000004079.

3. Hamedi Sangsari A, Sadr-Eshkevari P, Al-Dam A, et al. Surgically assisted rapid palatomaxillary expansion with or without pterygomaxillary disjunction: a systematic review and meta-analysis. J Oral Maxillofac Surg 2016;74(2):338-348. DOI: 10.1016/j.joms.2015.06.161.

4. Sygouros $A$, Motro $M$, Ugurlu $F$, et al. Surgically assisted rapid maxillary expansion. An evaluation of different surgical techniques and their effect on maxillary dentoskeletal complex based on cone-beam computed tomography. Preliminary report. Orthod $\mathrm{Fr}$ 2014;85(2):175-187. DOI: 10.1051/orthodfr/2014005.

5. Liberati A, Altman DG, Tetzlaff J, et al. The prisma statement for reporting systematic reviews and meta-analyses of studies that evaluate health care interventions: explanation and elaboration. J Clin Epidemiol 2009;62:1-34. DOI: 10.1016/j.jclinepi.2009. 06.006.

6. Cochrane Handbook for Systematic Reviews of Interventions Version 5.1.0 [updated March 2011], In: Higgins JPT, Green S The Cochrane Collaboration; 2011. Available at: https://handbook-5-1.cochrane. org/. Accessed June 26, 2019.

7. DerSimonian R, Laird N. Meta-analysis in clinical trials revisited. Contemp Clin Trials 2015;45(Pt A):139-145.
8. Higgins JP, Thompson SG. Quantifying heterogeneity in a metaanalysis. Stat Med 2002;21(11):1539-1558. DOI: 10.1002/sim.1186.

9. Egger M, Davey Smith G, Schneider M, et al. Bias in meta-analysis detected by a simple, graphical test. BMJ 1997;315(7109):629-634. DOI: 10.1136/bmj.315.7109.629.

10. Ferraro-Bezerra M, Tavares RN, de Medeiros JR, et al. Effects of pterygomaxillary separation on skeletal and dental changes after surgically assisted rapid maxillary expansion: a single-center, doubleblind, randomized clinical trial. J Oral Maxillofac Surg 2018;76(4): 844-853. DOI: 10.1016/j.joms.2017.08.032.

11. Laudemann K, Santo G, Revilla C, et al. Assessment of surgically assisted rapid maxillary expansion regarding pterygomaxillary disjunction using thin volume-rendering technique: in variance analysis and in reliability, accuracy, and validity. J Oral Maxillofac Surg 2011;69(10):2631-2643. DOI: 10.1016/j.joms.2010.12.007.

12. Zandi M, Miresmaeili A, Heidari A, et al. The necessity of pterygomaxillary disjunction in surgically assisted rapid maxillary expansion: a short-term, double-blind, historical controlled clinical trial. J Craniomaxillofac Surg 2016;44(9):1181-1186. DOI: 10.1016/j. jcms.2016.04.026.

13. Laudemann K, Petruchin O, Mack MG, et al. Evaluation of surgically assisted rapid maxillary expansion with or without pterygomaxillary disjunction based upon preoperative and post-expansion 3D computed tomography data. Oral Maxillofac Surg 2009;13(3):159-169. DOI: 10.1007/s10006-009-0167-3.

14. Pereira MD, de Abreu RA, Prado GP, et al. Strategies for surgically assisted rapid maxillary expansion according to the region of transverse maxillary deficiency. Int J Oral Maxillofac Surg 2012;41(9):1127-1130. DOI: 10.1016/j.ijom.2012.03.028.

15. Blæhr TL, Mommaerts MY, Kjellerup AD, et al. Surgically assisted rapid maxillary expansion with bone-borne vs tooth-borne distraction appliances-a systematic review. Int J Oral Maxillofac Surg 2019;48(4):492-501. DOI: 10.1016/j.ijom.2018.12.010.

16. Rômulo de Medeiros J, Ferraro Bezerra M, Gurgel Costa FW, et al. Does pterygomaxillary disjunction in surgically assisted rapid maxillary expansion influence upper airway volume? A prospective study using dolphin imaging 3D. Int J Oral Maxillofac Surg 2017;46(9):1094-1101. DOI: 10.1016/j.ijom.2017.04.010.

17. Vasconcelos BCE, Caubi AF, Dias E, et al. Surgically assisted rapid maxillary expasion: a preliminar study. Braz J Otorhinolaryngol 2006;72(4):457-461. DOI: 10.1016/S1808-8694(15)30990-3. 